\title{
Quantum phase transitions in a spin ladder with long-range couplings
}

\author{
Junpeng Cao, ${ }^{1}$ Gang Xiong, ${ }^{2,3}$ X.C. Xie, ${ }^{4,3}$ X.R. Wang, ${ }^{5}$ and Yupeng Wang*1,3 \\ ${ }^{1}$ Institute of Physics, Chinese Academy of Sciences, Beijing 100080, People's Republic of China \\ ${ }^{2}$ Physics Department, Beijing Normal University, Beijing 100875, People's Republic of China \\ ${ }^{3}$ International Center for Quantum Structures, Chinese Academy of Sciences, Beijing 100080, People's Republic of China \\ ${ }^{4}$ Physics Department, Oklahoma State University, Stillwater, OK 74078 \\ ${ }^{5}$ Physics Department, The Hong Kong University of Science and Technology, Hong Kong SAR, People's Republic of China
}

\begin{abstract}
An integrable model with rich ground state phases is hard to come by. In this Letter, an integrable spin ladder with pure long-range Heisenberg couplings is proposed and solved via the asymptotic Bethe ansatz method. Numerical solutions of the Bethe ansatz equation show that with the variation of the coupling range parameter $\alpha$, quantum phase transitions exist for both ferromagnetic $(J=-1)$ and anti-ferromagnetic $(J=1)$ intra-chain couplings. For $J=-1$, depending on $\alpha$, the ground state may be ferromagnetic, Takhatajian-Babujian spin liquid, or a frustrated spin liquid. For $J=1$, a quantum phase transition from the Heisenberg spin liquid to an exotic spin liquid occurs at a critical $\alpha$. The phase boundaries are determined numerically.
\end{abstract}

PACS numbers: 75.10.Jm, 75.30.Kz, 75.40.Cx

Competing effect between quantum phases of condensed matters is a relevant issue for a variety of important physical phenomena such as the colossal magnetoresistance $(\mathrm{CMR})$ in manganites and non-Fermi liquid behaviors in heavy fermion compounds. The CMR originates from the phase transition tuned by magnetic field between two delicately balanced and competing phases with rather different resistivities[1]. Non-Fermi liquid behaviors observed in several heavy fermion compounds may attribute to the quantum critical effect at the quantum phase boundaries. In addition, the low-dimensional spin systems may show fascinating competing behaviors among the long-range ordered, short-range ordered and disorder spin liquid phases and have recently attracted a lot of attentions because of the experimental realization in some quasi-one-dimensional materials $[2,3]$. A well known example is the zigzag spin lader or the $J_{1}-J_{2}$ spin chain, which may have gapless ground state with algebraically decaying spin correlations or gapped degenerate dimerized ground states $[4,5]$. The $J_{1}<0$, $J_{2}>0$ case was considered by Bader and Schilling [6] to study the competition between antiferromagnetic (AF) and ferromagnetic (FM) phases and found the sufficient and necessary conditions for FM ground state. A relevant experimental realization is the competing AF-FM orders induced by external field, temperature and other perturbations in $\mathrm{GdBaCO}_{2} \mathrm{O}_{5.5}$ single crystals[7].

As exact solutions of some two-dimensional statistical models play an important role to understand the phase transitions, solutions of quantum integrable models may provide a powerful starting point to understand the competing effect between delicately balanced quantum phases. However, the integrable model with rich ground state phases is still rare. In this Letter, we construct an integrable spin ladder model, which describes two coupled Inozemtsev spin chains[8]. The model is solved in the framework of the asymptotic Bethe ansatz method
[9]. It is shown that with the variation of the coupling range, there exist several ground state phases. The phase boundaries are determined via numerical analysis of the Bethe ansatz equation. This model therefore provides an outstanding example to study the quantum phase transitions in the low-dimensional spin systems.

The Hamiltonian of our ladder model reads

$H=-\frac{J^{\prime}}{2} \sum_{j \neq l, \beta=1,2} \frac{\vec{\sigma}_{\beta j} \cdot \vec{\sigma}_{\beta l}-1}{\sinh ^{2} \alpha(j-l)}+\frac{J^{\prime}}{2} \sum_{j \neq l} \frac{\vec{\sigma}_{1 j} \cdot \vec{\sigma}_{2 l}-1}{\cosh ^{2} \alpha(j-l)}$,

where $J^{\prime}=J \sinh ^{2} \alpha ; J= \pm 1$ and $\alpha$ the coupling constant which measures the interaction range of the spins; $j, l \in \mathbf{Z}$ and are odd for $\beta=1$ (chain 1 ) and even for $\beta=2$ (chain 2); $\vec{\sigma}_{\beta j}$ the Pauli matrix on $j$-th site in chain $\beta$. The quantum phase transitions in the present model are obvious by studying some limiting cases of the system for both $J= \pm 1$. 1) $J=1$ case: When $\alpha \rightarrow 0$, the system reduces to two decoupled FM Haldane-Shastry (HS) spin chains $[10,11]$ while when $\alpha \rightarrow \infty$, the system approaches to the AF Heisenberg spin chain. These two limiting cases possess rather different ground state properties and therefore quantum phase transition must occur when $\alpha$ goes from zero to infinity. 2) $J=-1$ case: When $\alpha \rightarrow 0$, the system decouples to two AF HS chains while when $\alpha \rightarrow \infty$, the system reduces to the FM Heisenberg chain, also indicating the existence of some quantum phase transition at a finite $\alpha$.

By introducing $a=\pi i / 2+\alpha$, the Hamiltonian (1) can be rewritten as

$$
H=-\frac{J^{\prime}}{2} \sum_{j \neq l} \frac{\vec{\sigma}_{j} \cdot \vec{\sigma}_{l}-1}{\sinh ^{2} a(j-l)}
$$

where $j, l$ take integer values. The above Hamiltonian is just the Inozemtsev quantum spin chain[8] but with a complex coupling constant $a$. However, the physics of the present model is quite different from the original Inozemtsev model for they indeed have rather different physical 
contents. We shall solve our model with Sutherland's asymptotic Bethe ansatz method[9]. The central point of the asymptotic Bethe ansatz is that for integrable models, the many-body scattering matrices can be factorized into the product of two-body scattering matrices and the Bethe ansatz equation (BAE) can be obtained by imposing the asymptotic periodic boundary conditions in the wave functions. The integrability of the system can be shown by constructing the following Dunkl operators [12]

$$
D_{j}=\sum_{k \neq j}[\operatorname{coth} a(j-k)-\operatorname{sign}(j-k)] P_{j k},
$$

where $\operatorname{sign}(j-k)$ is the sign function and $P_{j k}$ are the permutation operators. $P_{j k}$ obey the following laws:

$$
\begin{aligned}
& P_{j k}=P_{k j}=P_{j k}^{\dagger}, \quad P_{j k}^{2}=1, \\
& P_{j k} A_{k}=A_{j} P_{j k}, \quad P_{j k} A_{l}=A_{l} P_{j k}, \quad l \neq j, k,
\end{aligned}
$$

where $j, k \in \mathbf{Z}$ and $A_{j}$ are arbitrary many-body operators. Using these laws, one can prove that the Dunkl operators commute with each other. A complete set of conserved quantities of the system can be defined as $I_{n}=\sum_{j}\left(D_{j}\right)^{n}$. It is easy to demonstrate that these quantities are mutually commutative and commutative with the Hamiltonian by replacing $\left(\vec{\sigma}_{j} \cdot \vec{\sigma}_{l}+1\right) / 2$ in Eq.(2) with $P_{j l}$. Therefore, the system has an infinite number of conserved quantities and is integrable. Detailed demonstration of the integrability of this kind of models can be found in[13].

To derive the exact solution of the Hamiltonian, we note that the FM vacuum state $\left|\Omega>=\otimes_{j \in \mathbf{z}}\right| \uparrow>_{j}$ is an eigenstate of the system with zero eigenvalue. Since the $z$-component of total spin is a conserved quantity, the eigenstates of the system can be classified by the number of flipped spins $M$ over the FM vacuum state. A general state with $M$ flipped spins is

$$
\left|\psi>=\sum_{n_{1} \neq \cdots \neq n_{M}} \psi\left(n_{1}, \cdots, n_{M}\right) \sigma_{n_{1}}^{-} \cdots \sigma_{n_{M}}^{-}\right| \Omega>,
$$

where $\sigma_{n_{j}}^{-}$is the spin lowering operator on $n_{j}$-th site and $n_{j} \in \mathbf{Z}$. Assume the $M$-magnon wave function is

$$
\begin{aligned}
& \psi\left(n_{1}, \ldots n_{M}\right)=\prod_{\mu \neq \nu}^{M} \sum_{P \in \pi_{M}, m \in D^{M}} \frac{(-1)^{P} A_{\{m\}}(\{k\})}{\sinh a\left(n_{\mu}-n_{\nu}\right)} \\
& \quad \times \exp \left[\sum_{\lambda=1}^{M}\left(i k_{P \lambda} n_{\lambda}+a\left(2 m_{P \lambda}-M+1\right) n_{\lambda}\right)\right],
\end{aligned}
$$

where $P$ is the permutation of the permutation group $\pi_{M}, D^{M}$ is the hypercube in $\mathbf{Z}^{M},\{k\}$ are the quasimomenta of magnons for scattering states and $A_{\{m\}}(\{k\})$ are the coefficients. The eigenvalue equation, $H \mid \psi>=$ $E_{M} \mid \psi>$ can be written in terms of the $M$-magnon wave function as $[8,14]$

$$
\begin{aligned}
& \sum_{\rho=1}^{M} \sum_{s \in \mathbf{Z}_{[n]}} V\left(n_{\rho}-s\right) \psi\left(n_{1}, \ldots, n_{\rho-1}, s, n_{\rho+1}, \ldots, n_{M}\right) \\
= & -\psi\left(n_{1}, \ldots, n_{M}\right)\left[\sum_{\rho \neq \gamma}^{M} V\left(n_{\rho}-n_{\gamma}\right)+\frac{E_{M}}{J^{\prime}}-M E_{0}\right],
\end{aligned}
$$

where the notation $\mathbf{Z}_{[n]}$ is used for the variety $\mathbf{Z}$ $\left(n_{1}, \ldots, n_{M}\right), V(x)=\sinh ^{-2} a x$ and $E_{0}=\sum_{n \neq 0} V(n)$. Following the method proposed by Inozemtsev [8], we obtain the eigenenergy of this state as

$$
\begin{aligned}
E_{M}= & -2 J \sinh ^{2} \alpha \sum_{j=1}^{M} \sum_{n \neq 0} \frac{\cos 2 n k_{j}-1}{\sinh ^{2}(2 n \alpha)} \\
& +2 J \sinh ^{2} \alpha \sum_{j=1}^{M} \sum_{n} \frac{\cos (2 n+1) k_{j}-1}{\cosh ^{2}(2 n+1) \alpha} .
\end{aligned}
$$

In the framework of the asymptotic Bethe ansatz method[9], the BAE can be obtained by imposing the periodic boundary condition on the asymptotic form of the wave function, $\psi\left(n_{2}, \cdots, n_{M}, n_{1}+L\right)=\psi\left(n_{1}, \cdots, n_{M}\right)$ for $L \rightarrow \infty$. In our case it reads

$$
\lim _{L \rightarrow \infty} e^{i k_{j} L}=\prod_{l=1, \neq j}^{M} S^{-1}\left(k_{j}-k_{l}\right), \quad j=1,2, \cdots, M,
$$

where $S\left(k_{j}-k_{l}\right)$ is the two-body scattering matrix

$$
S\left(k_{j}-k_{l}\right)=-\frac{1-i\left[\frac{k_{j}}{\pi} \zeta(\pi)-\zeta\left(k_{j}\right)-\frac{k_{l}}{\pi} \zeta(\pi)+\zeta\left(k_{l}\right)\right]}{1+i\left[\frac{k_{j}}{\pi} \zeta(\pi)-\zeta\left(k_{j}\right)-\frac{k_{l}}{\pi} \zeta(\pi)+\zeta\left(k_{l}\right)\right]},
$$

where $\zeta(x)$ is the Weierstrass function with double quasiperiodicities $\omega_{1}=2 \pi$ and $\omega_{2}=\pi-2 i \alpha$. The scattering matrix is determined by the asymptotic form of the two-magnon wavefunction.

Below we solve the BAE numerically. According to the Lieb-Mattis theorem[15], a bi-partite spin system has either a spin singlet ground state or a ferromagnetic ground state. To determine the phase boundary between the FM state and a spin singlet state, an easy way is to study the excitation spectrum of the FM state. If all excitation modes carry positive energy, the FM state is the ground state while if any mode's energy becomes negative (soft mode), the ground state must be some kind of spin singlet state.

i)The $J=-1$ case. In this case, the system resembles features of the Bader-Shilling model[6]. When $\alpha$ is large, the interchain FM coupling is dominant and the ground state should be FM, while for small $\alpha$ the intrachain AF coupling is dominant and the ground state should be a spin singlet. Thus a quantum phase transition from an FM ground state to an AF spin liquid 
ground state is expected at some critical $\alpha$ value $\alpha_{c r}$. As shown in Fig. 1, the one-magnon spectrum is always positive for $\alpha>0.757$ while part of the spectrum becomes negative when $\alpha<0.757$. However, to determine the ground state exactly we need also to examine other kinds of solutions of the BAE, say, the string solutions or the bound states of magnons[16]. For the two-magnon bound state excitations, let us denote the momenta of two magnons as $k_{1}$ and $k_{2}$, respectively. In the case of a bound state, $k_{1}$ and $k_{2}$ should be a pair of complex conjugate numbers as $k_{1}=k_{1}^{\prime}+i k_{2}^{\prime}$ and $k_{2}=k_{1}^{\prime}-i k_{2}^{\prime}$, where $k_{1}^{\prime}$ and $k_{2}^{\prime}$ are real. The vanishing of the wave function when the distance between the two down spins tends to infinity requires

$$
2 \zeta(\pi) k_{2}^{\prime} / \pi+i\left[\zeta\left(k_{1}^{\prime}+i k_{2}^{\prime}\right)-\zeta\left(k_{1}^{\prime}-i k_{2}^{\prime}\right)\right]=1 .
$$

The origin $k_{1}^{\prime}=k_{2}^{\prime}=0$ is the order-one singular point of the Weierstrass $\zeta$ function and should be considered directly from the original BAE. One can easily show that

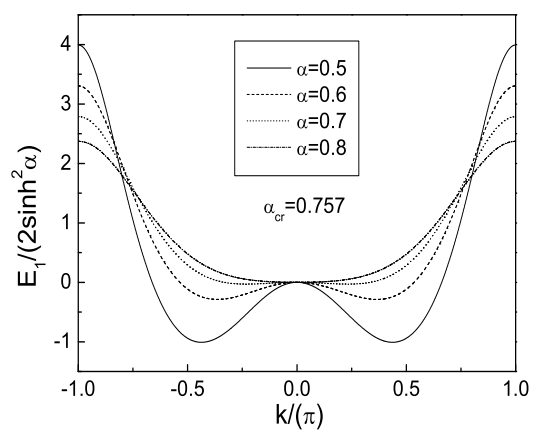

FIG. 1: The one-magnon energy spectrum curve. All the energies are positive for $\alpha>0.757$, while the spectrum has negative regions when $\alpha<0.757$. When $\alpha \rightarrow 0$, the curve has two minimums at $k= \pm \pi / 2$ and two maximums at $k= \pm \pi$.

$k_{2}^{\prime}=k_{1}^{\prime}=0$ is a solution of the BAE. The two-magnon bound state energy spectrum is shown in Fig. 2. One can see that if $\alpha>0.776$, all the two-magnon bound states have non-negative energy. It should be noted that for $0.757<\alpha<0.776$ a part of the two-magon spectrum can be negative when the one-magnon spectrum is positive due to the competition of intrachain $\mathrm{AF}$ and interchain FM couplings. In the case of $m$-magnon bound state, $k_{j}$ should satisfy the string hypothesis $f\left(k_{j}\right)=\lambda_{m}+i(m+$ $1-2 j) / 2, j=1,2, \cdots, m$, where $\lambda_{m}$, the center of the $m$ string, is a real number. We check numerically that the $3,4,5,6$-string energies also turn to positive at $\alpha=0.776$. In fact, $\epsilon_{n}(k) / n$ is an increasing function of $n$, where $\epsilon_{n}(k)$ is the energy of the $n$-string. Thus we expect that for $\alpha>0.776$ all the excited spectrums are positive and the FM vacuum state is the ground state and quantum phase transition occurs at $\alpha_{c r 1}=0.776$. Let us consider
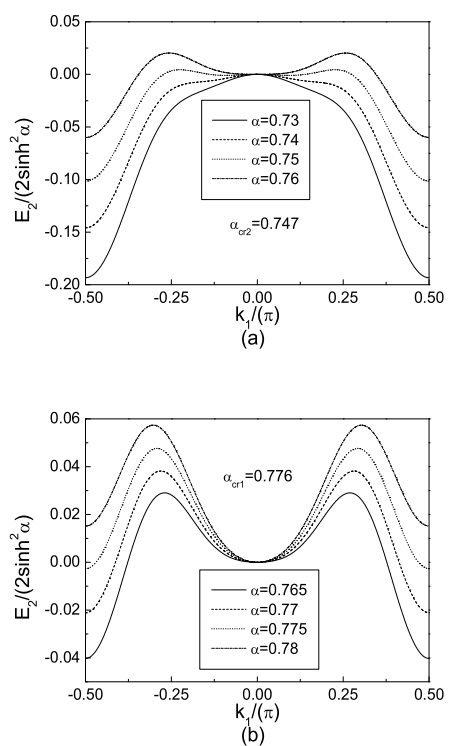

FIG. 2: The two-magnon bound state energy spectrum curve. (a) All the energies are negative for $\alpha<0.747$. With increasing $\alpha$, positive energy regions begin to emerge. (b) All the energies are positive for $\alpha>0.776$. With decreasing $\alpha$, regions of negative energy appear.

further the structure of the spin liquid ground state in $\alpha<\alpha_{c r 1}$. When $\alpha<\alpha_{c r 2}=0.747$ all the energy of twomagnon bound states are negative. Numerical solutions show that the state consisting of all two-magnon bound states has the lowest energy by comparing its energy to those of other string solutions. This spin liquid state here is very similar to that of the spin-1 Takhtajian-Babujian model [17], we call this phase the T-B phase. Because the 2-string at $k=0$ carries zero energy, this spin liquid is gapless. In the region $0.747<\alpha<0.776$, part of the two-magnon state becomes negative. However, only with these modes one can not get a spin singlet states. Thus the ground state in this region must consist of different kinds of $m$-magnon bound states and its structure is very complicated due to strong frustration. We call this phase the frustrated phase. From Bader-schilling's sufficient and necessary conditions for the existence of a FM ground state [6], we approximately get $\alpha_{c r} \approx 0.881$, which is reasonably close to our exact result $\alpha_{c r 2}=0.776$.

ii) The $J=1$ case. The one-magnon energy is always negative at $k= \pm \pi$ for all $\alpha$. Thus the excitation spectrum always has a negative energy region and the FM state is not the ground state. However, there also exists a quantum phase transition between two spin liquid phases controlled by the value of $\alpha$. For $\alpha>0.757$, all the onemagnon states have negative energies. With the same method used for the usual Heisenberg spin chain, one can prove that the state consisting of all one-magnon states occupied, i.e., the gapless Heisenberg spin liquid state has 
the lowest energy by studying the hole and string excitations of this state[16]. Taking the logarithm of Eq.(6), we have

$$
\frac{I_{j}}{L}=\frac{\pi-k_{j}}{2 \pi}-\frac{1}{\pi L} \sum_{l \neq j}^{M} \arctan \left[f\left(k_{j}\right)-f\left(k_{l}\right)\right],
$$

where $I_{j}$ is an integer or half-integer and $f(k)=$ $k \zeta(\pi) / \pi-\zeta(k)$. In the thermodynamic limit, the density of the quasi-particle is defined as $\sigma(\lambda)=d Z(\lambda) / d \lambda$, where $\lambda \equiv f(k), Z\left(\lambda_{j}\right) \equiv I_{j} / L$. Taking the derivative of Eq.(8), we have

$$
\sigma(\lambda)=\frac{\mu^{\prime}(\lambda)}{2 \pi}-\int_{-\infty}^{\infty} A\left(\lambda-\lambda^{\prime}\right) \sigma_{\infty}\left(\lambda^{\prime}\right) d \lambda^{\prime},
$$

where $A(\lambda)=\left[\pi\left(1+\lambda^{2}\right)\right]^{-1}, \mu^{\prime}(\lambda)$ is the derivative of $\mu(\lambda), \mu(\lambda) \equiv \pi-k$. A simple calculation gives $M / L=$ $\int \sigma(\lambda) d \lambda=1 / 2$, indicating a total spin zero state. When $\alpha<0.757$, the energy spectrum of one-magnon states has positive region and the Heisenberg spin liquid ground state is no longer stable. By checking the string solutions numerically we find that an $M=L / 2$-magnon bound state at $k=0$ has the lowest energy and should be the ground state in this case. A classical picture of this ground state is that all the spins in one chain take the same direction and are bounded, while all the spins in the other chain take the opposite direction and are also bounded, i.e., the Ising spin-density-wave state if we treat the system as a single spin chain. The low-lying excited states are those breaking the bound state. The lowest excited state consists of an $(M-2)$-magnon bound state and 2 magnons. According to the Bethe ansatz equation, the allowed momentum value of the magnons tend to zero in the thermodynamic limit and the energies of magnons are zero, indicating that this exotic spin liquid is gapless. Therefore, a quantum phase transition from a Heisenberg spin liquid phase to an exotic Ising-spin-density-wave-like liquid state phase is expected at $\alpha_{c r}=0.757$. A direct diagonalization of an eight-site system confirms the above results.
In summary, an integrable zigzag spin ladder with long range competing interactions is constructed. The exact spectrum is derived via the asymptotic Bethe ansatz method. Numerical analysis showed that quantum phase transitions exist for both $J= \pm 1$. This model provides an elegant spin system from which we can study quantum phase transitions exactly.

X.R. Wang is supported by UGC, Hong Kong. Y. Wang is supported by the NSF of China. He also thanks Physics Department of HKUST for their hospitality during his visit. X.C. Xie is supported by US DOE with grant number DE-FG02-04ER46124.

*Email: yupeng@aphy.iphy.ac.cn

[1] For a review, see E. Dagotto, T. Hotta, and A. Moreo, Phys. Rep. 344, 1 (2001).

[2] E. Dagotto and T.M. Rice, Science 271, 618 (1996).

[3] G. Chaboussant et al., Phys. Rev. Lett. 80, 2713 (1998).

[4] F.D.M. Haldane, Phys. Rev. B. 25, 4925 (1982).

[5] B.S. Shastry and B. Sutherland, Phys. Rev. Lett. 47,964 (1981).

[6] H.P. Bader and R. Schilling, Phys. Rev. B 19, 3556 (1979); 21, 1304 (1980).

[7] A.A. Taskin et al., Phys. Rev. Lett. 90, 227201 (2003).

[8] V.I. Inozemtsev, J. Stat. Phys. 59, 1143 (1990); Commun. Math. Phys. 148, 359 (1992).

[9] B. Sutherland, Rocky Mtn. J. of Math. 8, 431 (1978); B. Sutherland and B.S. Shastry, Phys. Rev. Lett. 71, 5 (1993).

[10] F.D.M. Haldane, Phys. Rev. Lett. 60, 635 (1988).

[11] B.S. Shastry, Phys. Rev. Lett. 60, 639 (1988).

[12] A.P. Polychronakos, Phys. Rev. Lett. 69, 703 (1992).

[13] D. Bernard et al., J. Phys. A 26, 5219 (1993).

14] O.A. Chalykh and A.P. Veselov, Commun. Math. Phys. 126, 597 (1990).

[15] E.H. Lieb, and D.C. Mattis, Phys. Rev. 125, 164 (1962).

[16] M. Takhashi, Thermodynamics of One-dimensional Solvable Models, (Cambridge University Press, 1999).

[17] L.A. Takhtajian, Phys. Lett. A. 87, 479 (1982); H.M. Babujian, Phys. Lett. A. 90, 479 (1982). 